\title{
Denosumab: an investigational drug for the management of postmenopausal osteoporosis
}

\author{
E Michael Lewiecki \\ New Mexico Clinical Research \& \\ Osteoporosis Center, Albuquerque, \\ New Mexico, USA
}

\begin{abstract}
Denosumab (AMG 162) is an investigational fully human monoclonal antibody with a high affinity and specificity for receptor activator of nuclear factor- $\mathrm{BB}$ ligand (RANKL), a cytokine member of the tumor necrosis factor family. RANKL, the principal mediator of osteoclastic bone resorption, plays a major role in the pathogenesis of postmenopausal osteoporosis and other skeletal disorders associated with bone loss. Denosumab inhibits the action of RANKL, thereby reducing the differentiation, activity, and survival of osteoclasts, and lowering the rate of bone resorption. Clinical trials have shown that denosumab increases bone mineral density (BMD) and reduces bone turnover in postmenopausal women with low BMD. Studies to evaluate the fracture risk benefit and long-term safety of denosumab in women with postmenopausal osteoporosis (PMO) are ongoing. Denosumab is a potential treatment for PMO and other skeletal disorders.
\end{abstract}

Keywords: osteoporosis, treatment, denosumab, AMG 162, RANKL, OPG

\section{Introduction}

Osteoporosis is a disease characterized by low bone mineral density (BMD) and poor bone quality that reduces bone strength and increases the risk of low-trauma fractures (Klibanski et al 2001). It is a worldwide public health problem with serious consequences in terms of personal suffering and costs to society (Autier et al 2000; Burge et al 2007). About $30 \%$ of postmenopausal Caucasian women have osteoporosis (Melton 1995), with a lifetime fracture risk of $40 \%$ (Melton et al 1992). Fractures of the hip and spine are associated with increased morbidity and mortality (Cooper 1997; Center et al 1999). Although clinical tools to diagnosis osteoporosis and predict fracture risk are readily available (Kanis et al 2005), patients who are at risk for fracture are often not recognized or treated (Solomon et al 2005). Even patients with previous fractures, who are at very high risk of future fracture (Johnell et al 2004), are commonly not evaluated or treated (Feldstein et al 2003). When patients are started on a drug for the treatment for osteoporosis, compliance to therapy is poor (McCombs et al 2004), with many studies showing less than $50 \%$ of patients still taking drug one year after it has been prescribed (Cramer et al 2007). Patients who are compliant to therapy have a greater increase in BMD (Yood et al 2003), lower fracture risk (Caro et al 2004), and reduced healthcare costs (McCombs et al 2004) compared to those who are not compliant.

Drugs for the treatment of osteoporosis may be classified as antiresorptive (also called anti-catabolic) or anabolic (Riggs and Parfitt 2005), depending on their predominant effect on bone remodeling. Estrogens, bisphosphonates (eg, alendronate, risedronate, ibandronate, zoledronate), calcitonin, and raloxifene are antiresorptive agents that strengthen bone and reduce fracture risk. By decreasing bone turnover, these drugs stabilize or increase BMD by filling in the remodeling space and prolonging secondary mineralization, maintaining bone microarchitecture, reducing trabecular perforation, and decreasing cortical porosity. 
Teriparatide, recombinant human parathyroid hormone (PTH) 1-34, is an anabolic or bone-forming drug that strengthens bone and reduces fracture risk by increasing bone formation, which is associated with an increase in bone size and possibly restoration or formation of new trabecular microarchitectural elements. Strontium ranelate, which is approved in some countries for the treatment of osteoporosis, appears to have both antiresorptive and anabolic properties. While all of the approved drugs play a useful role in the management of osteoporosis, each has its limitations. Oral bisphosphonates have complex administration requirements (fasting, ingestion with plain water only, post-dose fasting) and may cause upper gastrointestinal symptoms. Intravenous (iv) bisphosphonates must be given by trained staff in a physician's office or infusion center. Estrogen and raloxifene are associated with non-skeletal risks (eg, thromboembolic events), as well as non-skeletal benefits, such as reduction in hot flashes with estrogen and decreased risk of invasive breast cancer with raloxifene. Nasal calcitonin may be the best tolerated of all agents, but can cause nasal irritation in some patients. Teriparatide involves daily self-administered subcutaneous injection and is the most expensive of all approved drugs. The US Surgeon General has identified poor adherence to therapy as a major obstacle in the treatment of osteoporosis, and suggested that less frequent dosing and simplified drug administration be considered as a potential means to improve adherence (US Department of Health and Human Services 2004).

Denosumab (AMG 162; Amgen Inc., Thousand Oaks, CA, USA) is an investigational drug with a novel mechanism of action that is being developed for use in the management of postmenopausal osteoporosis (PMO). Administered as a subcutaneous injection (SC) at intervals as long as 6 months, it addresses some of the concerns raised by the US Surgeon General regarding poor adherence to therapy and may overcome some of the limitations of currently available drugs. Denosumab is now in phase 3 clinical trials for the prevention and treatment of postmenopausal osteoporosis and other skeletal diseases associated with bone loss.

\section{Denosumab structure and mechanism of action}

Denosumab is a fully human monoclonal antibody $\left(\mathrm{IgG}_{2}\right.$ immunoglobulin isotype) with a high affinity and specificity for human receptor activator of nuclear factor- $\kappa \mathrm{B}$ ligand (RANKL). It is manufactured with XenoMouse ${ }^{\circledR}$ (Abgenix, Inc., Fremont, CA, USA) technology using a mouse strain engineered to produce a wide range of human antibodies in the absence of mouse antibodies. Denosumab is a potent inhibitor of osteoclastic bone resorption. By binding to
RANKL in a manner similar to native osteoprotegerin (OPG), denosumab prevents the interaction of RANKL and RANK. This reduces the differentiation, activity, and survival of osteoclasts, thereby inhibiting bone resorption.

\section{Physiology of the adult skeleton}

The adult human skeleton is a metabolically active organ system that is continually renewed through the process of bone remodeling (bone turnover). Packets of bone collagen and mineral are resorbed (removed) and formed (replaced) in small discreet cavities. These cavities form pits or trenches on the surfaces of trabecular (cancellous) bone and tunnels or canals within cortical bone in Haversian systems. Bone remodeling is essential for mineral homeostasis and maintenance of bone viability, including the replacement of apoptotic osteocytes and repair of microdamage. In healthy adults with stable bone mass, the rate of bone turnover is moderate with resorption and formation being of approximately equal magnitude. Excessive remodeling (high bone turnover) diminishes bone strength independently of BMD, primarily because excessive numbers of cavities act as "stress risers"weakened areas of bone that may be a source of microcracks. High bone turnover also weakens bone due to loss of BMD associated with microarchitectural deterioration of bone tissue (eg, trabecular thinning, trabecular perforation, loss of trabecular connectivity, cortical thinning, and increased cortical porosity) and diminished mineralization. When bone resorption is greater than bone formation, as typically occurs in early postmenopausal estrogen-deficient women, there is a net loss of bone over time. If unchecked, this can eventually result in low bone mass (osteopenia) or osteoporosis. In physiological states and with most metabolic bone diseases there is "coupling" of bone resorption and bone formation, ie, a sequence of events where osteoclastic bone resorption is predictably followed by osteoblastic bone formation. As bone resorption rises or falls, so does bone formation. When formation fails to keep up with resorption, bone loss occurs, and when formation exceeds resorption, there is a net increase in bone mass. The cellular and humoral factors that regulate bone remodeling are the focus of great interest, with advances in this field providing a better understanding of the origins of skeletal health and disease, and suggesting possible targets for pharmacological interventions to treat skeletal diseases.

\section{Regulation of bone remodeling}

While there are numerous systemic and local factors involved in the regulation of bone remodeling, the principal final mediator of osteoclastic bone resorption is RANKL, 
a cytokine member of the tumor necrosis factor (TNF) superfamily (Boyle et al 2003). RANKL is a 316 amino acid transmembrane protein that is highly expressed by osteoblasts and $\mathrm{T}$ cells (Collin-Osdoby 2004). It is also found in two biologically active soluble forms derived by cleavage from the osteoblast cell surface or secreted from T cells. Receptor activator of nuclear factor- $\mathrm{\kappa B}$ (RANK), located on the cell membrane of osteoclasts and pre-osteoclasts (Hsu et al 1999), is a 616 amino acid transmembrane receptor for RANKL. RANKL-RANK binding stimulates the differentiation, activity, and survival of osteoclasts, resulting in increased bone resorption (Lacey et al 1998). Soluble RANKL injected into mice results in a measurable increase in bone resorption within 60 minutes (Burgess et al 1999). Osteoprotegerin (OPG), a 380 amino acid member of the TNF receptor superfamily, is a soluble non-signaling "decoy receptor" for RANKL. By binding to RANKL and preventing its interaction with RANK, OPG inhibits osteoclast differentiation (Simonet et al 1997; Lacey et al 1998), activity (Mosekilde et al 1991; Burgess et al 1999), attachment to the bone surface (O'Brien et al 2000, 2001) and survival (Lacey et al 2000), thereby reducing bone resorption (Simonet et al 1997). An increase in RANKL in proportion to OPG is associated with the development of postmenopausal osteoporosis and other skeletal disorders that include multiple myeloma, metastatic bone disease, treatment-related bone loss, rheumatoid arthritis, and Paget's disease of bone (Lacey et al 1998; Boyle et al 2003; Hofbauer and Schoppet 2004). RANKL expression on osteoblasts is upregulated by such diverse signals as PTH, 1,25-dihydroxyvitamin D $\left(1,25-[\mathrm{OH}]_{2}-\mathrm{D}\right)$, calcium, glucocorticoids, prostaglandin E2, inteleukin (IL)-1 $\alpha$, IL-6, IL-11, and IL-17, and downregulated by transforming growth factor (TGF)- $\beta$. OPG is increased by signals that include 1,25-(OH) - D, calcium, IL-1 $\alpha$, IL-6, IL-11, IL-17, estrogen, TGF- $\beta$, and bone morphogenetic protein (BMP)-2, and decreased by continuous administration of PTH, glucocorticoids, prostaglandin E2, and insulin-like growth factor 1 (Collin-Osdoby 2004). In patients with primary hyperparathyroidism, an elevated RANKL:OPG ratio was observed using assays for RANKL and OPG mRNA on transiliac bone biopsy, with a significant decrease after parathyroidectomy (Stilgren et al 2004). Intermittent PTH therapy in women with glucocorticoid-induced osteoporosis modulates serum levels of RANKL and OPG (Buxton et al 2004), suggesting that the RANKL/RANK/OPG signaling pathway participates in the therapeutic effects of this agent. Flow cytometry of bone marrow cells of pre- and postmenopausal women showed upregulation of RANKL after menopause (Eghbali-Fatourechi et al 2003), consistent with RANKL playing a role in high bone turnover and bone loss commonly associated with this population.

\section{RANKL inhibition and immune function}

RANKL is expressed not only by osteoblasts and preosteoblasts, by also by endothelial cells, stromal cells, primitive mesenchymal cells surrounding cartilage, chondrocytes, activated T lymphocytes, and immature CD4/CD8 thymocytes. RANK is expressed on the surface of chondrocytes, mammary gland epithelial cells, trophoblast cells, dendritic cells, and mature T cells, as well as on the surface of osteoclasts. OPG is produced and released by cells that include osteoblasts, dendritic cells, and lymphocytes, and OPG is expressed in organs that include the heart, lung, spleen, thymus, kidney and intestine (Neumann et al 2005). Evidence is accumulating that bone remodeling is modulated through a complex interaction of osteoclasts and osteoblasts with immune cells (eg, T and B lymphocytes, dendritic cells), cytokines (eg, interleukin-1, interleukin-6, tumor necrosis factor- $\alpha$ ), and circulating hormones (eg, vitamin D, parathyroid hormone, testosterone, leptin) (Clowes et al 2005). A local or systemic imbalance in these factors may result in bone loss, as commonly occurs in many chronic inflammatory diseases. It has been demonstrated that activated $\mathrm{T}$ cells directly stimulate osteoclastogenesis through RANKL (Kong et al 1999a). It is a fundamental concept of osteoimmunology that RANKL produced by activated $\mathrm{T}$ and $\mathrm{B}$ lymphocytes increases bone resorption through its interaction with osteoclast precursors and that RANKL also prolongs the survival of dendritic cells, thereby increasing $\mathrm{T}$ cell activity (Wong et al 1997; Josien et al 1999; Xing et al 2005).

The intimate relationship of bone remodeling with RANKL/RANK/OPG signaling and regulators of immune function has raised concern that agents that inhibit RANKL could have adverse effects on the immune response to infection or malignancy (Whyte 2006). Preclinical studies suggest that the development of an intact immune system is dependent on the RANKL/RANK/OPG pathway. RANK knockout mice, for example, have a deficiency of splenic B cells and impaired lymph node organogenesis (Dougall et al 1999; Kong et al 1999b). In contrast, the RANKL/ RANK/OPG system appears to have a non-essential role in immune function for adults who already have a fully developed immune system. Studies in OPG transgenic rats and mice that overexpress OPG to levels 100-fold greater than wild type animals have enhanced understanding of 
the prenatal role of RANKL/RANK/OPG signaling on the skeleton and the immune system. These animals have normal lymph node formation and normal immune systems (Stolina et al 2007), suggesting that profound inhibition of RANKL prenatally is not harmful to the development of an intact immune system in rodents, and that only small quantities of RANKL are sufficient for lymph node formation.

\section{Pharmacokinetics and metabolism of denosumab}

The pharmacokinetics of denosumab are nonlinear with dose, as has been observed with other fully human monoclonal antibodies. Although the absorption, bioavailability, distribution, and elimination of denosumab have not been well defined, studies of other therapeutic monoclonal IgG antibodies given by the sc route provide insight into what is likely with denosumab. Studies with similar IgG antibodies suggest that SC denosumab is probably absorbed via the lymphatic system, with subsequent drainage of lymph fluid into the vascular system (Lobo et al 2004). Bioavailability is probably in the range of $50 \%-100 \%$, with a distribution that is about the same as the plasma volume (Lobo et al 2004; Tang et al 2004). Clearance is probably by the reticuloendothelial system (Lobo et al 2004). No significant amount of circulating denosumab appears to be filtered and excreted by the kidneys. In a phase 1 dose-escalation trial of denosumab given as a single sc dose to healthy postmenopausal women, 3 phases were reported: a prolonged absorption phase with maximum serum concentration $\left(\mathrm{C}_{\max }\right)$ at 5-21 days post-dose, increasing as the dose increased; a prolonged $\beta$-phase, with serum half-life as long as 32 days with the maximum dose; and a rapid terminal phase when serum concentration fell below $1000 \mathrm{ng} / \mathrm{mL}$ (Bekker et al 2004). When denosumab is given as a $60 \mathrm{mg}$ sc dose every 6 months, currently being studied in phase 3 clinical trials for the prevention and treatment of $\mathrm{PMO}$, the median time to maximum concentration $\left(\mathrm{T}_{\max }\right)$ after the first dose is 26 days (Peterson et al 2005). Denosumab is a more potent antiresorptive agent with a longer duration of antiresorptive effect than equivalent doses of OPG-Fc (a fusion molecule of recombinant OPG with the Fc fragment of IgG), evaluated in early clinical trials of RANKL inhibition (Bekker et al 2001; Body et al 2003). The long duration of denosumab activity can be attributed to its long half-life as well as effects in inhibiting osteoclast recruitment and activity. Preclinical models have suggested that the delay in osteoclast recovery observed after withdrawal of RANKL inhibition is due to the time necessary for osteoclasts to regenerate from precursor cells (Lacey et al 2000; Capparelli et al 2003). A feature distinguishing denosumab from the bisphosphonates is the rapid reversibility of its antiresorptive effect once it has been eliminated and osteoclast regeneration has occurred, since it is not incorporated into bone matrix, as are the bisphosphonates.

\section{Preclinical studies of RANKL inhibition}

RANKL is the principal final mediator of osteoclastic bone resorption. This has been well documented in preclinical studies evaluating the role of the RANKL/RANK/OPG signaling pathway in the regulation of bone remodeling. In cell cultures, RANKL induces osteoclast-like cell formation (Matsuzaki et al 1998). Recombinant OPG inhibits osteoclast differentiation in a dose-dependent manner (Simonet et al 1997). Osteotropic hormones and cytokines are have been observed to regulate expression of RANKL and OPG in human-derived osteoblast cell lines (Hofbauer et al 1999). OPG knockout mice develop osteoporosis and fragility fractures (Bucay et al 1998). RANKL knockout mice (Kong et al 1999b) and RANK knockout mice (Li et al 2000) have osteopetrosis associated with a total absence of osteoclasts.

Because of the high specificity of denosumab for human RANKL, most preclinical studies of RANKL inhibition in mice and rats must use agents other than denosumab. The compounds most commonly used are fusion molecules of recombinant OPG or RANK and the Fc fragment of $\mathrm{IgG}$, called OPG-Fc or RANK-Fc, respectively. Recombinant OPG prevents bone loss in ovariectomized (OVX) rats (Simonet et al 1997). Recombinant OPG decreases osteoclast differentiation in normal mice, resulting in non-lethal osteopetrosis (Simonet et al 1997). In mice with low BMD that overexpress TNF- $\alpha$, recombinant OPG prevents bone loss (Schett et al 2003). RANKL inhibition increases bone mineralization and improves mechanical strength in the femur of young male rats (Ross et al 2001). In a study of aged OVX rats, a combination of recombinant rat OPG and PTH increased BMD more than either agent alone (Kostenuik et al 2001). Intact 4- to 5-year-old male cynomolgus monkeys treated with denosumab were observed to have an increase in bone mass and improvement in bone strength with threepoint bending of the femur and compression testing of lumbar vertebral bodies (Atkinson et al 2005). Preclinical models of rheumatoid arthritis (Redlich et al 2002), ovariectomy (Kostenuik et al 2004), multiple myeloma (Vanderkerken et al 2003), and inflammatory bowel disease (Byrne et al 2005) have demonstrated skeletal benefit with RANKL inhibition. 
Knockin technology has recently been used to replace the 5th exon of the murine RANKL gene with a hybrid exon containing the coding region for the 5 th exon of the human RANKL gene (plus a murine non-coding region), resulting in genetically engineered mice expressing a chimeric form of RANKL (human/murine) that is bound and neutralized by denosumab (Kostenuik et al 2005). This non-primate model has subsequently provided a platform for evaluating the skeletal effects of denosumab in variety of circumstances with potential human applications (Ferrari et al 2007; Hofbauer et al 2007).

\section{Clinical studies of RANKL inhibition: efficacy OPG-Fc in healthy postmenopausal women}

The first study of RANKL inhibition in humans was conducted with OPG-Fc. This was a phase 1 randomized, double-blind, placebo-controlled, sequential dose escalation study in healthy postmenopausal women (Bekker et al 2001). Each of the 52 subjects in this study received a single SC dose of OPG-Fc $0.1,0.3,1.0,3.0 \mathrm{mg} / \mathrm{kg}$, or placebo. All subjects received physical exams and laboratory studies that included hematology, blood chemistries, coagulation studies, immunoglobulins, T cell enumeration (CD3, CD4, CD8), bone turnover markers, and neutralizing antibodies to OPG. The highest dose $(3.0 \mathrm{mg} / \mathrm{kg})$ resulted in a decrease of urinary N-telopeptide (NTX), a marker of bone resorption, within 12 hours of dosing. Maximum suppression of NTX was approximately $80 \%$ below baseline after 3 days, with a subsequent slow rise to $14 \%$ below baseline at 6 weeks. There was a decline in bone-specific alkaline phosphatase (BSAP), a marker of bone formation, that began about 3 weeks after dosing, reaching a low point of about $30 \%$ below baseline at 6 weeks. This pattern of rapid and profound suppression of bone resorption with slower and less pronounced suppression of bone formation is similar to what was observed in preclinical studies. This study showed that a single dose of OPG-Fc caused a rapid, reversible, and dose-dependent suppression of bone resorption. OPG-Fc was not associated with serious adverse events, dropouts due to adverse events, or neutralizing antibodies to OPG. The most common adverse event was a mild injection site reaction. The findings of this study provided support for further clinical development of RANKL inhibition with denosumab, an agent having greater antiresorptive potency and longer duration of action than OPG-Fc.

\section{Denosumab in healthy postmenopausal women}

A phase 1 single-dose, randomized, double-blind, placebocontrolled, dose escalation study of denosumab was conducted in healthy postmenopausal women (Bekker et al 2004). There were 49 subjects in this study, each of whom was given a single SC dose of denosumab 0.01, 0.03, 0.1, $0.3,1.0$, or $3.0 \mathrm{mg} / \mathrm{kg}$, or placebo. They were followed for as long as 6 months in all cohorts and 9 months in the cohorts receiving the 3 highest doses. Drug safety, tolerability, pharmacokinetics, and effects on bone turnover markers were evaluated. Suppression of urinary NTX was dose-dependent, rapid (as early as 12 hours, the first time of measurement), profound (as much as $84 \%$ reduction from baseline), sustained (as long as 6 months), and reversible (increasing at the end of the monitoring period). Suppression of BSAP occurred later and was less pronounced than NTX. Subsequent clinical investigation of denosumab was undertaken in the study described below.

\section{Denosumab in postmenopausal women with low BMD}

A phase 2 randomized, placebo-controlled, dose-ranging study was conducted to evaluate the efficacy and safety of denosumab in postmenopausal women with low BMD, having a lumbar spine T-score of -1.8 to -4.0 , or total hip or femoral neck T-score of -1.8 to -3.5 (McClung et al 2006; Lewiecki et al 2007; Miller et al 2008). Study subjects were randomized to 9 groups of 41-54 women, with each group assigned to receive $\mathrm{SC}$ denosumab 6,14 , or $30 \mathrm{mg}$ every 3 months or 14 , 60,100 , or $210 \mathrm{mg}$ every 6 months; open-label alendronate 70 mg weekly; or placebo. All study subjects received daily oral supplements of calcium (1000 mg) and vitamin D (400 IU). The mean age of randomized subjects was 63 years, with all groups well matched for demographics and baseline characteristics. The ethnicity classification was $85 \%$ white, $11 \%$ hispanic, and 3\% black. The primary endpoint was the percentage change in lumbar spine (L1-L4) BMD at 12 months compared to baseline. Percentage change from baseline in BMD at the total hip, femoral neck, distal one-third radius, total body minus head were also assessed. Bone turnover was evaluated by measurement of serum CTX, urinary NTX, and BSAP. A total of 412 women were enrolled in the study, with $369(90 \%)$ completing 12 months of treatment.

At 12 months, treatment with denosumab was associated with a lumbar spine BMD increase of $3.0 \%$ to $6.7 \%$, depending on dose and dosing interval, while there was a 
$0.8 \%$ loss with placebo ( $\mathrm{p}<0.001$ for difference) (McClung et al 2006). At the total hip, there was a BMD increase of $1.9 \%$ to $3.6 \%$ from baseline, while a $0.6 \%$ loss was observed in the placebo group ( $\mathrm{p}<0.001$ for difference). At the distal one-third radius, there was a BMD increase of $0.4 \%-1.3 \%$ from baseline with denosumab, compared to a $2.0 \%$ loss with placebo ( $\mathrm{p}<0.001$ for difference). In an exploratory comparison with open-label alendronate, denosumab $30 \mathrm{mg}$ every 3 months and $60 \mathrm{mg}$ every 6 months were associated with a similar BMD responses at the lumbar spine and apparently greater BMD increases at the total hip and distal one-third radius. Denosumab-treated patients had a decrease in serum CTX compared to placebo $(p<0.001)$ that was observed as early as 3 days, the first scheduled time of CTX measurement. Serum CTX suppression reached a maximum mean decrease of $87.8 \%$ compared to $4.2 \%$ with placebo. The effects on urinary NTX were similar to serum CTX. Denosumab was associated with suppression of BSAP compared to placebo $(\mathrm{p}<0.001)$ that was delayed by about 1 month compared to CTX. The suppression of bone turnover was dose-dependent, rapid, sustained, and reversible. The data suggested that denosumab $60 \mathrm{mg}$ every 6 months and $30 \mathrm{mg}$ every 3 months were associated with maximum therapeutic benefit and minimal exposure dose.

A prespecified exploratory analysis evaluated the efficacy and safety of denosumab after 24 months or treatment (Lewiecki et al 2007). Outcome measures included BMD at the lumbar spine, total hip, distal one-third radius, and total body, as well as bone turnover markers and safety. Of the original 412 women randomized, 337 (81.8\%) completed the 24 month study. BMD increases at the lumbar spine at 24 months ranged from $4.13 \%$ to $8.89 \%$ with denosumab compared to a $1.18 \%$ decrease with placebo $(\mathrm{p}<0.001$ for all doses denosumab vs. placebo). The percentage changes in lumbar spine BMD from baseline for all doses of denosumab doses were greater than for placebo from month 3 to month 24 ( $\mathrm{p}<0.001)$. At 24 months, all doses of denosumab were associated with BMD increases at the total hip, one-third radius, and total body compared to placebo ( $\mathrm{p}<0.001$ ). Compared to open label alendronate, BMD increases with denosumab at all four skeletal sites were similar or greater, except with the denosumab dose of $14 \mathrm{mg}$ every 6 months, for which the BMD change at the lumbar spine was less than with alendronate. With the denosumab dose of $60 \mathrm{mg}$ every 6 months (the dose being studied in a phase 3 fracture prevention trial), the BMD changes from 12 months to 24 months were evaluated. The BMD increase during the second 12 months of the study was $2.75 \%$ at the lumbar spine $(\mathrm{p}<0.001)$ and $1.50 \%$ at the total hip $(\mathrm{p}<0.001)$, with numerically greater but statistically insignificant changes in BMD at the distal one-third radius and total body.

\section{Ongoing clinical trials with denosumab}

Phase 3 trials are currently underway to evaluate the safety and efficacy of denosumab in reducing vertebral fracture risk in women with PMO, prevention of bone loss in postmenopausal women with low BMD (osteopenia), prevention of bone loss in women with non-metastatic breast cancer on aromatase inhibitor therapy, and prevention of bone loss in men with nonmetastatic prostate cancer on androgen deprivation therapy. Ongoing phase 2 trials include the evaluation of denosumab in patients with rheumatoid arthritis, bisphosphonate-naïve patients with breast cancer, and patients receiving intravenous bisphosphonates for advanced cancer metastatic to bone.

An interim analysis of treatment with 5 dosing regimens of denosumab in 255 bisphosphonate naïve patients with breast cancer-related bone metastases has been reported (Lipton et al 2007). In this phase 2, randomized, active-controlled, multi-center, multidose, parallel group study, patients were randomized to receive SC denosumab 30, 120, or $180 \mathrm{mg}$ every 4 weeks, 60 or $180 \mathrm{mg}$ every 12 weeks, or openlabel iv bisphosphonate (zoledronic acid, pamidronate, or ibandronate at the physician's discretions) every 4 weeks. After 13 weeks of treatment, the median percentage reduction in urinary $\mathrm{NTX} /$ creatinine ratio $(\mathrm{uNTX} / \mathrm{Cr}$ ) was $71 \%$ for the pooled denosumab groups and $79 \%$ for the IV bisphosphonate group. Overall, 74\% of denosumab-treated patients achieved more than a $65 \%$ reduction in uNTX/Cr compared to $63 \%$ for the bisphosphonate-treated patients. The greatest overall median suppression of $\mathrm{uNTX} / \mathrm{Cr}$ at 13 weeks was observed with denosumab $120 \mathrm{mg}$ every 4 weeks.

\section{Safety and tolerability of denosumab}

In the phase 1 study of denosumab in healthy postmenopausal women, there were no drug-related serious adverse events and no discontinuations from the study due to adverse events (Bekker et al 2004). Mild transient dose-dependent decreases in albumin-adjusted serum calcium and corresponding increases in serum intact parathyroid (iPTH) levels were observed. Infectious adverse events were similar with denosumab (38\% in the combination of all treatment groups) and placebo (33\%). Two subjects treated with denosumab (1.0 and $3.0 \mathrm{mg} / \mathrm{kg}$ ) had mild injection site reactions. No denosumab-associated changes in white blood cell (WBC) counts, T, B, or NK cell counts (CD3, CD4, CD8, CD20, 
CD56), immunoglobulins, or coagulation parameters were reported.

The 12-month report of the phase 2 study of denosumab in women with low BMD described a small, transient, asymptomatic, non-dose dependent decrease in the mean albumin-adjusted serum calcium in patients treated with denosumab compared to placebo and alendronate (McClung et al 2006). The mean serum iPTH increased with denosumab and alendronate compared to placebo, with a return toward baseline values over time. There were no significant differences in the percentage of subjects having adverse events with denosumab, placebo, and alendronate, with the exception of dyspepsia being most common with open-label alendronate. Two subjects receiving denosumab $100 \mathrm{mg}$ every 6 months developed transient non-neutralizing denosumab-binding antibodies. In a subset of denosumab-treated subjects who had immune function evaluated, there were no clinically meaningful changes in white blood cell counts, $\mathrm{T}$ cell, B cell or NK cell (CD3, CD4, CD8, CD19, and CD16/56) enumeration by flow cytometry at 1-year (Cohen et al 2005). There was no evidence that denosumab was associated with an increased risk of infection, malignancy, over-suppression of bone turnover, or impaired fracture healing. There were no reported cases of osteonecrosis of the jaw with denosumab.

Over the first 24 months of the phase 2 trial, adverse events continued to be generally similar in the placebo, denosumab, and alendronate groups (Lewiecki et al 2007). There were 6 cases $(1.9 \%)$ of serious adverse events of infections in the denosumab group ( 2 cases of diverticulitis, 3 cases of pneumonia, and 1 case of labyrinthitis) compared to none in the placebo group or open label alendronate group. There was 1 death, caused by gastric cancer, in the denosumab group, and none in the placebo or alendronate groups. Discontinuation rates over the first 24 months were $2.2 \%$ for placebo, 2.9\% for denosumab, and 6.4\% for alendronate. No neutralizing antibodies to denosumab were observed in the first 24 months of treatment.

In the 13 week interim analysis of safety data in the phase 2 trial of denosumab in women with breast cancer-related bone metastases, $8 \%$ of denosumab-treated patients experienced transient asymptomatic decreases in serum calcium compared to $5 \%$ of IV bisphosphonate-treated patients (Lipton et al 2007). Serious adverse events in the study were similar for those treated with denosumab and bisphosphonates, with none of the serious adverse events attributed to denosumab or iv bisphosphonate. There was no apparent increase in the rate of infections or cardiovascular events in denosumab-treated patients, and no reports of osteonecrosis of the jaw. Injection-site reactions were reported in 3\% of denosumab-treated patients and no IV bisphosphonatetreated patients.

There is no evidence that RANKL inhibition in humans has adverse effects on immune function (Bekker et al 2001, 2004; McClung et al 2006).

\section{Potential role of denosumab in the management of PMO}

As denosumab in currently available for investigational use only, and studies phase 3 trials evaluating prevention of bone loss in postmenopausal women with low bone mass and reduction of fracture risk in women with PMO have not been completed, it is purely speculative to consider its potential role in clinical practice. However, the published data to date suggest that it may have some advantages over therapeutic agents that are now approved. The SQ method of dosing is likely to expand the number of physicians willing to administer the drug compared to iv bisphosphonates, which are primarily given at infusion centers and by physicians specializing in osteoporosis. The long dosing interval of 6 months assures adherence to therapy for that length of time- a potential advantage over oral agents administered daily, weekly, or monthly.

If the phase 3 trials demonstrate efficacy that is similar to the bisphosphonates, with a good safety profile, then the target population may largely depend on cost- an unknown value at this time. If the cost is sufficiently low, denosumab might be considered as "first-line" therapy for PMO. If the cost is similar to branded oral or IV bisphosphonates, as is more likely to be the case, then drug selection for an individual patient may depend on availability, previous experience (if any) with bisphosphonates, and convenience of administration.

Although it is unlikely that a large head-to-head clinical trial comparing denosumab and bisphosphonates, with fractures as the primary endpoint, will ever be conducted, studies comparing surrogates of bone strength may show some differences between these drug classes. If denosumab has a greater effect on cortical bone than bisphosphonates, as suggested by differences in BMD response at the radius compared to alendronate in a phase 2 clinical trial (Lewiecki et al 2007), then it could have some theoretical advantages in the treatment of patients at high risk for non-vertebral fractures.

Finally, preclinical studies showing an additive effect of OPG and PTH on BMD suggest a potential role for combination therapy with denosumab and anabolic agents. Further study is needed to test this hypothesis. 


\section{Summary}

Denosumab is an investigational fully human monoclonal antibody with high affinity and specificity to RANKL, the principal mediator of osteoclastic bone resorption. It suppresses bone resorption in a rapid, sustained, and reversible manner. Denosumab treatment, given as a sc injection with a dosing frequency as long as 6 months, increases BMD and reduces bone turnover in postmenopausal women with low BMD. Reported adverse events in a phase 2 clinical trial in women with low BMD were similar to placebo and openlabel alendronate. Denosumab is a promising therapeutic agent for the management of postmenopausal osteoporosis and other skeletal diseases associated with bone loss. Phase 3 trials to evaluate the efficacy of denosumab to reduce the risk of fractures in women with PMO or prevent bone loss in individuals with other skeletal disorders are ongoing.

\section{Note}

Amgen released a press release on July 25, 2008 (http://www. amgen.com/media/media_pr_detail.jsp?releaseID=1179628) announcing the results of a pivotal, three-year, international, phase 3 study of denosumab in women with osteoporosis.

\section{Disclosures}

Dr. Lewiecki has received financial support or owned personal investments in the following categories during the past one year: Grant/Research Support (principal investigator, funding to New Mexico Clinical Research \& Osteoporosis Center) Amgen, Eli Lilly, GSK, Merck, Novartis, Pfizer, Procter \& Gamble, Roche, Sanofi Aventis, and Wyeth.

Other support: Amgen - scientific advisory board; Eli Lilly - scientific advisory board, speakers' bureau; Merck - scientific advisory board; Novartis - scientific advisory board, speakers' bureau; Procter \& Gamble/Sanofi Aventis - scientific advisory board; Roche/GSK - scientific advisory board, speakers' bureau; Servier-scientific advisory board; Upsher-Smith - scientific advisory board; Wyeth scientific advisory board.

Direct stock shareholder: General Electric, Procter \& Gamble, Teva.

\section{References}

Atkinson JE, Cranmer P, Saunders T, et al. 2005. Denosumab (AMG 162), a fully human RANKL antibody, increases bone mass and bone strength in cynomolgus monkeys. J Bone Miner Res, 20(Suppl 1):S294.

Autier P, Haentjens P, Bentin J, et al. 2000. Costs induced by hip fractures: a prospective controlled study in Belgium. Belgian Hip Fracture Study Group. Osteoporos Int, 11:373-80.

Bekker, PJ, Holloway, D, Nakanishi, A, et al. 2001. The effect of a single dose of osteoprotegerin in postmenopausal women. J Bone Miner Res, $16: 348-60$.
Bekker PJ, Holloway DL, Rasmussen AS, et al. 2004. A single-dose placebo-controlled study of AMG 162, a fully human monoclonal antibody to RANKL, in postmenopausal women. $J$ Bone Miner Res, 19:1059-66.

Body JJ, Greipp P, Coleman RE, et al. 2003. A phase I study of AMGN0007, a recombinant osteoprotegerin construct, in patients with multiple myeloma or breast carcinoma related bone metastases. Cancer, 97(3 Suppl):887-92.

Boyle WJ, Simonet WS, Lacey DL. 2003. Osteoclast differentiation and activation. Nature, 423:337-42.

Bucay N, Sarosi I, Dunstan CR, et al. 1998. osteoprotegerin-deficient mice develop early onset osteoporosis and arterial calcification. Genes Dev, 12:1260-8.

Burge R, Dawson-Hughes B, Solomon DH, et al. 2007. Incidence and economic burden of osteoporosis-related fractures in the United States, 2005-2025. Journal of Bone and Mineral Research, 22:465-75.

Burgess TL, Qian Y, Kaufman S, et al. 1999. The ligand for osteoprotegerin (OPGL) directly activates mature osteoclasts. $J$ Cell Biol, 145:527-38.

Buxton EC, Yao W, Lane NE. 2004. Changes in serum receptor activator of nuclear factor-kappaB ligand, osteoprotegerin, and interleukin-6 levels in patients with glucocorticoid-induced osteoporosis treated with human parathyroid hormone (1-34). J Clin Endocrinol Metab, 89:3332-6.

Byrne FR, Morony S, Warmington K, et al. 2005. CD4+CD45RBHi T cell transfer induced colitis in mice is accompanied by osteopenia which is treatable with recombinant human osteoprotegerin. Gut, 54:78-86.

Capparelli C, Morony S, Warmington K, et al. 2003. Sustained antiresorptive effects after a single treatment with human recombinant osteoprotegerin (OPG): a pharmacodynamic and pharmacokinetic analysis in rats. $J$ Bone Miner Res, 18:852-8.

Caro JJ, Ishak KJ, Huybrechts KF, et al. 2004. The impact of compliance with osteoporosis therapy on fracture rates in actual practice. Osteoporos Int, 15:1003-8.

Center JR, Nguyen TV, Schneider D, et al. 1999. Mortality after all major types of osteoporotic fracture in men and women: an observational study. Lancet, 353:878-82.

Clowes JA, Riggs BL, Khosla S. 2005. The role of the immune system in the pathophysiology of osteoporosis. Immunol Rev, 208:207-27.

Cohen SB, Lewiecki EM, Liu Y, et al. 2005. Phase 2 study of denosumab (AMG 162) in postmenopausal women: subanalyses and supplemental safety. J Bone Miner Res, 20(Suppl 1):S295.

Collin-Osdoby P. 2004. Regulation of vascular calcification by osteoclast regulatory factors RANKL and osteoprotegerin. Circ Res, 95:1046-57.

Cooper C. 1997. The crippling consequences of fractures and their impact on quality of life. Am J Med, 103:12S-9S.

Cramer JA, Gold DT, Silverman SL, et al. 2007. A systematic review of persistence and compliance with bisphosphonates for osteoporosis. Osteoporos Int, 18:1023-31.

Dougall WC, Glaccum M, Charrier K, et al. 1999. RANK is essential for osteoclast and lymph node development. Genes Dev, 13(18):2412-24.

Eghbali-Fatourechi G, Khosla S, Sanyal A, et al. 2003. Role of RANK ligand in mediating increased bone resorption in early postmenopausal women. J Clin Invest, 111:1221-30.

Feldstein A, Elmer PJ, Orwoll E, et al. 2003. Bone mineral density measurement and treatment for osteoporosis in older individuals with fractures - A gap in evidence-based practice guideline implementation. Arch Intern Med, 163:2165-72.

Ferrari SL, Pierroz D, Bonnet N, et al. 2007. A comparison of denosumab and alendronate in combination with PTH in ovariectomized knockin mice expressing humanized RANKL. J Bone Miner Res, 22(Suppl 1):S37.

Hofbauer LC, Khosla S, Dunstan CR, et al. 1999. Estrogen stimulates gene expression and protein production of osteoprotegerin in human osteoblastic cells. Endocrinology, 140:4367-70.

Hofbauer LC, Schoppet, M. 2004. Clinical implications of the osteoprotegerin/RANKL/RANK system for bone and vascular diseases. JAMA, 292:490-5. 
Hofbauer LC, Zeitz U, Schoppet M, et al. 2007. RANK Ligand inhibition by denosumab prevents cortical bone loss in a murine model of glucocorticoid-induced osteoporosis. J Bone Miner Res, 22(Suppl 1):S4.

Hsu H, Lacey DL, Dunstan CR, et al. 1999. Tumor necrosis factor receptor family member RANK mediates osteoclast differentiation and activation induced by osteoprotegerin ligand. Proc Natl Acad Sci US A, 96:3540-5.

Johnell O, Kanis JA, Oden A, et al. 2004. Fracture risk following an osteoporotic fracture. Osteoporos Int, 15:175-9.

Josien R, Wong BR, Li HL, et al. 1999. TRANCE, a TNF family member, is differentially expressed on $\mathrm{T}$ cell subsets and induces cytokine production in dendritic cells. $J$ Immunol, 162:2562-8.

Kanis JA, Borgstrom F, De Laet C, et al. 2005. Assessment of fracture risk. Osteoporos Int, 16:581-9.

Klibanski A, Adams-Campbell L, Bassford T, et al. 2001. Osteoporosis prevention, diagnosis, and therapy. JAMA, 285:785-95.

Kong YY, Feige U, Sarosi I, et al. 1999a. Activated T cells regulate bone loss and joint destruction in adjuvant arthritis through osteoprotegerin ligand. Nature, 402:304-9.

Kong YY, Yoshida H, Sarosi I, et al. 1999b. OPGL is a key regulator of osteoclastogenesis, lymphocyte development and lymph-node organogenesis. Nature, 397:315-23.

Kostenuik PJ, Bolon B, Morony S, et al. 2004. Gene therapy with human recombinant osteoprotegerin reverses established osteopenia in ovariectomized mice. Bone, 34:656-64.

Kostenuik PJ, Capparelli C, Morony S, et al. 2001. OPG and PTH-(1-34) have additive effects on bone density and mechanical strength in osteopenic ovariectomized rats. Endocrinology, 142:4295-304.

Kostenuik PJ, Warmington K, Grisanti M, et al. 2005. RANKL inhibition with (denosumab) AMG 162, a fully human MAb, causes sustaned suppression of bone resorption and increased BMD in knockin mice expressing humanized RANKL. J Bone Miner Res, 20(Suppl 1):S261.

Lacey DL, Tan HL, Lu J, et al. 2000. Osteoprotegerin ligand modulates murine osteoclast survival in vitro and in vivo. Am J Pathol, $157: 435-48$.

Lacey DL, Timms E, Tan HL, et al. 1998. Osteoprotegerin ligand is a cytokine that regulates osteoclast differentiation and activation. Cell, 93:165-76.

Lewiecki EM, Miller PD, McClung MR, et al. 2007. Two-year treatment with denosumab (AMG 162) in a randomized phase 2 study of postmenopausal women with low BMD. J Bone Miner Res, 22:1832-41.

Li J, Sarosi I, Yan XQ, et al. 2000. RANK is the intrinsic hematopoietic cell surface receptor that controls osteoclastogenesis and regulation of bone mass and calcium metabolism. Proc Natl Acad Sci U S A, 97:1566-71.

Lipton A, Steger GG, Figueroa J, et al. 2007. Randomized active-controlled phase ii study of denosumab efficacy and safety in patients with breast cancer-related bone metastases. J Clin Oncol, 25:4431-7.

Lobo, ED, Hansen, RJ, and Balthasar, JP. 2004. Antibody pharmacokinetics and pharmacodynamics. J Pharm Sci, 93(11):2645-68.

Matsuzaki K, Udagawa N, Takahashi N, et al. 1998. Osteoclast differentiation factor (ODF) induces osteoclast-like cell formation in human peripheral blood mononuclear cell cultures. Biochem Biophys Res Commun, 246:199-204.

McClung MR, Lewiecki EM, Cohen SB, et al. 2006. Denosumab in postmenopausal women with low bone mineral density. $N$ Engl $J$ Med, 354:821-31.

McCombs JS, Thiebaud P, Laughlin-Miley C, et al. 2004. Compliance with drug therapies for the treatment and prevention of osteoporosis. Maturitas, 48:271-87.

Melton LJ III. 1995. How many women have osteoporosis now? J Bone Miner Res, 10:175-7.

Melton LJ III, Chrischilles EA, Cooper C, et al. 1992. Perspective. How many women have osteoporosis? J Bone Miner Res, 7:1005-10.

Miller PD, Bolognese MA, Lewiecki EM, et al. 2008. Effect of denosumab on bone density and turnover in postmenopausal women with low bone mass after long-term continued, discontinued, and restarting of therapy: A randomized blinded phase 2 clinical trial. Bone, 43:222-9.
Mosekilde L, Sogaard CH, Danielsen CC, et al. 1991. The anabolic effects of human parathyroid hormone (hPTH) on rat vertebral body mass are also reflected in the quality of bone, assessed by biomechanical testing: a comparison study between $\mathrm{hPTH}-(1-34)$ and $\mathrm{hPTH}-(1-84)$. Endocrinology, 129:421-8.

Neumann E, Gay S, Muller-Ladner U. 2005. The RANK/RANKL/ osteoprotegerin system in rheumatoid arthritis: new insights from animal models. Arthritis Rheum, 52:2960-7.

O'Brien EA, Williams JH, Marshall, MJ. 2000. Osteoprotegerin ligand regulates osteoclast adherence to the bone surface in mouse calvaria. Biochem Biophys Res Commun, 274:281-90.

O'Brien EA, Williams JH, Marshall, MJ. 2001. Osteoprotegerin is produced when prostaglandin synthesis is inhibited causing osteoclasts to detach from the surface of mouse parietal bone and attach to the endocranial membrane. Bone, 28:208-14.

Peterson MC, Stouch BJ, Martin SW, et al. 2005. The pharmacokinetics of denosumab (AMG 162) following various multiple subcutaneous dosing regimens in postmenopausal women with low bone mass. J Bone Miner Res, 20(Suppl 1):S293.

Redlich K, Hayer S, Maier A, et al. 2002. Tumor necrosis factor alphamediated joint destruction is inhibited by targeting osteoclasts with osteoprotegerin. Arthritis Rheum, 46:785-92.

Riggs BL, Parfitt, AM. 2005. Drugs used to treat osteoporosis: the critical need for a uniform nomenclature based on their action on bone remodeling. J Bone Miner Res, 20:177-84.

Ross AB, Bateman TA, Kostenuik PJ, et al. 2001. The effects of osteoprotegerin on the mechanical properties of rat bone. J Mater Sci Mater Med, 12:583-8.

Schett G, Redlich K, Hayer S, et al. 2003. Osteoprotegerin protects against generalized bone loss in tumor necrosis factor-transgenic mice. Arthritis Rheum, 48:2042-51.

Simonet WS, Lacey DL, Dunstan CR, et al. 1997. Osteoprotegerin: a novel secreted protein involved in the regulation of bone density. Cell, 89:309-19.

Solomon DH, Morris C, Cheng H, et al. 2005. Medication use patterns for osteoporosis: an assessment of guidelines, treatment rates, and quality improvement interventions. Mayo Clin Proc, 80:194-202.

Stilgren LS, Rettmer E, Eriksen EF, et al. 2004. Skeletal changes in osteoprotegerin and receptor activator of nuclear factor-kappab ligand mRNA levels in primary hyperparathyroidism: effect of parathyroidectomy and association with bone metabolism. Bone, 35:256-65.

Stolina M, Dwyer D, Morony S, et al. 2005. Rats and mice overexpressing soluble OPG have high bone mass but no alteration in immunological parameters or lymphocyte function. Arthritis Rheum, (52):S708.

Stolina M, Dwyer D, Ominsky MS, et al. 2007. Continuous RANKL inhibition in osteoprotegerin transgenic mice and rats suppresses bone resorption without impairing lymphorganogenesis or functional immune responses. J Immunol, 179:7497-505.

Tang L, Persky AM, Hochhaus G, et al. 2004. Pharmacokinetic aspects of biotechnology products. J Pharm Sci, 93:2184-204.

US Department of Health and Human Services. 2004. Bone Health and Osteoporosis: A Report of the Surgeon General. Rockville, MD, US Department of Health and Human Services, Office of the Surgeon General.

Vanderkerken K, De LE, Shipman C, et al. 2003. Recombinant osteoprotegerin decreases tumor burden and increases survival in a murine model of multiple myeloma. Cancer Res, 63:287-9.

Whyte MP. 2006. The long and the short of bone therapy. N Engl J Med, 354:860-3.

Wong BR, Josien R, Lee SY, et al. 1997. TRANCE (tumor necrosis factor [TNF]-related activation-induced cytokine), a new TNF family member predominantly expressed in T cells, is a dendritic cell-specific survival factor. $J$ Exp Med, 186:2075-80.

Xing L, Schwarz EM, Boyce, BF. 2005. Osteoclast precursors, RANKL/ RANK, and immunology. Immunol Rev, 208:19-29.

Yood RA, Emani S, Reed JI, et al. 2003. Compliance with pharmacologic therapy for osteoporosis. Osteoporos Int, 14:965-8. 
